Rev. Biol. Trop. 52(2): 355-362, 2004

www.ucr.ac.cr www.ots.ac.cr www.ots.duke.edu

\title{
Addendum to the Checklist of the helminth parasites of vertebrates in Costa Rica
}

\author{
Beatriz Rodríguez-Ortíz ${ }^{1}$, Luis García-Prieto², Jonathan Herrera-Vázquez ${ }^{1} \&$ \\ Gerardo Pérez-Ponce de León ${ }^{2}$ \\ 1 Laboratorio de Helmintología, Facultad de Microbiología, Universidad de Costa Rica, San José, Costa Rica, e-mail: \\ brodrigu@cariari.ac.ucr.cr; jonnathan@biologia.ucr.ac.cr \\ 2 Laboratorio de Helmintología, Instituto de Biología, UNAM. Ap. Postal 70-153. C.P. 04510, México D.F., e-mail: \\ ppdleon@servidor.unam.mx; gprieto@ibiologia.unam.mx
}

$$
\text { Received 22-II-2004. Corrected 01-III-2004. Accepted 01-III-2004. }
$$

\begin{abstract}
In this work, we present an addendum to the "Checklist of the helminth parasites of vertebrates in Costa Rica" with a parasite-host list as well as a host-parasite list. This addendum updates the available information on this group of parasites in Costa Rica, since very recently a new input has been made to describe the helminth fauna of vertebrates, particularly at the Área de Conservación de Guanacaste. In this paper, we add 33 records, representing 23 species. This raises the number of helminth species described in vertebrates from Costa Rica to 325 , represented by 89 species of digeneans, 23 of monogeneans, 63 of cestodes, 13 of acanthocephalans, and 137 of nematodes. In total, 133 species of vertebrates have been studied for helminths in Costa Rica (31 species of fishes, 7 amphibians, 18 reptiles, 40 birds, and 37 mammals). Currently, 67 species (20.6\%) have been recorded as new species from Costa Rica and most of them are endemic to particular regions. The Colección de Helmintos de Costa Rica, housed at the Universidad de Costa Rica (UCR), San José, is the depositary of only $23 \%$ of the species recorded so far; however this situation is changing and people recognizes.
\end{abstract}

Key Words: Helminths, Costa Rica, Biodiversity, Biological inventories, Parasites, Vertebrates.

Palabras Clave: Helmintos, Costa Rica, Biodiversidad, Inventarios biológicos, Parásitos, Vertebrados.

The checklist of helminth parasites of vertebrates in Costa Rica, published recently (Rodríguez-Ortiz et al. 2004), updated all the available information about helminth parasites of vertebrates in Costa Rica along the past 50 years. Notwithstanding, the inventory of all eukaryotic parasites of the species of vertebrates living within the Área de Conservación Guanacaste, Costa Rica, started in 1996 by Daniel R. Brooks and co-workers, has produced a great dealt of information in the last two years. For this reason, we present this addendum, in order to compile all this information and some other available records, all of them will be available electronically in 2004 at www.chcr.ucr.ac.cr.

\section{MATERIALS AND METHODS}

Refer to Rodríguez-Ortiz et al. (2004).

\section{RESULTS}

This addendum to the checklist contains records of 23 species included in 17 genera of helminths (9 digeneans, 1 acanthocephalan and 13 nematodes). 14 of them represent new species and 4 new genera. Helminths were reported in 25 species of vertebrates not previously studied in Costa Rica. Likewise, we add some taxonomic remarks made in recent publications on 5 species included in the original 
checklist. Next, we present the information for each species of helminth.

Symbology: $\mathrm{B}=$ bird; $\mathrm{M}=$ mammal; $\mathrm{R}=$ reptile; NA= information not available; $\mathrm{CHCR}$ refers to the Colección de Helmintos de Costa Rica, San José, Costa Rica; HWML to the Harold W. Manter Laboratory of Parasitology, University of Nebraska State Museum, Lincoln, Nebraska, U.S.A.; USNPC to the U.S. National Parasite Collection, Beltsville, Maryland, U.S.A.

\section{PLATYHELMINTHES}

Gegenbaur, 1859

Digenea (Van Beneden, 1858)

Brachylaimoidea

Joyeux and Foley, 1930

1. Bakkeius moragai Zamparo, Brooks and Causey, 2003

Gallbladder, intestine, Gymnopithys leucaspis (B), Estación San Gerardo, Guanacaste, Henicorhina leucosticta, Phaenostictus mcleannani, Thryothorus pleurostictus (B), Maritza and Sendero la Parcela, Guanacaste (Zamparo et al. 2003b). Specimens: CHCR, USNPC.

2. Pojmanskia riosae Zamparo, Brooks and Causey, 2003

Intestine, gallbladder, Thryothorus pleurostictus (B), Cafetal, Guanacaste, Caryothraustes poliogaster, Euphonia hirudinacea, Morococcyx erythropygius, Platyrinchus coronatus, Thryothorus nigricapillus, (B), Estación San Gerardo, Guanacaste (Zamparo et al. 2003b). Specimens: CHCR, USNPC.

Notes: Both genus were not assigned to any of the families within the Brachylaimoidea.

Brachycoeliidae (Looss, 1899) Johnston, 1912

3. Mesocoelium monas (Rudolphi, 1819) Teixeira de Freitas, 1958
Intestine, Norops limifrons (R), Hacienda La Suerte, Limón (Bursey and Goldberg 2003). Specimens: HWML.

Notes: Bursey and Goldberg (2003) refers the record of Mesocoelium travassosi realized by Caballero and BrenesMadrigal (1958) in toads from Costa Rica to the species $M$. monas. Both species were synonymyzed by Teixeira de Freitas (1963).

Brachylaimidae (Joyeux and Foley, 1930)

4. Tinamutrema canoae Zamparo, Brooks and Causey, 2003

Cloaca, Crypturellus cinnamomeus (B), Finca Jenny, Guanacaste (Zamparo et al. 2003a). Specimens: CHCR, USNPC.

Cyclocoeliidae

Kossack, 1911

5. Neohaematotrephus arayae Zamparo, Brooks, Causey and Rodríguez, 2003

Body cavity, Jacana spinosa (B), Laguna Los Jícaros, Guanacaste (Zamparo et al. 2003d). Specimens: CHCR, USNPC.

Macroderoididae

McMullen, 1937

6. Parallopharynx gonzalezi BrenesMadrigal, Arroyo-Sancho and MonteroGei, 1960

Intestine, Ctenosaura quinquecarinata (R), Cuajiniquil, Guanacaste (Zamparo et al. 2004b). Specimens: CHCR, USNPC.

7. P. matternae Zamparo, Brooks, Tkach and Rodríguez, 2004

Intestine, Basiliscus basiliscus (R), Río Tempisquito, Guanacaste (Zamparo et al. 2004b). Specimens: CHCR, USNPC.

Prosthogonimidae (Lühe, 1909)

Lahille, 1922

8. Whallwachsia illuminata Zamparo, Brooks and Causey, 2003 
Bile duct, intestine, Amazilia saucerrottei (B), Cafetal, Guanacaste, Tolomomyias sulphurescens (B), main entrance to Santa Rosa, Parcela Principe, Estación San Gerardo, Guanacaste (Zamparo et al. 2003c). Specimens: CHCR, USNPC.

\section{Urotrematidae}

Poche, 1926

9. Urotrema shirleyae Zamparo, Brooks, and Tkach, 2004

Intestine, Norops oxylophus (R), Santa Rosa, Guanacaste (Zamaparo et al., 2004a). Specimens: CHCR, USNPC.

\section{ACANTHOCEPHALA}

(Rudolphi, 1808)

Echinorhynchidae Cobbold, 1876

1. Acanthocephalus saurius Bursey and Goldberg, 2003

Intestino, Norops limifrons (R), Hacienda La Suerte, Limón (Bursey and Goldberg 2003). Specimens: HWML.

\section{NEMATODA}

(Rudolphi, 1808)

Acuariidae Seurat, 1913

1. Acuariidae gen. sp. (larvae)

Stomach wall, Norops limifrons (R), Hacienda La Suerte, Limón (Bursey and Goldberg 2003). Specimens: HWML.

2. Acuaria mayori Lent, Teixeira de Freitas and Proenca, 1945

Gizzard, Myiarchus nuttingi (B), Cafetal and Quebrada Costa Rica, Guanacaste (Zhang et al. 2003a). Specimens: USNPC.

3. A. wangi Zhang, Brooks and Causey, 2003 Gizzard, Gymnipithys leucaspis and Hylophylax naevioides (B), Estación San Gerardo, Guanacaste (Zhang et al. 2003a). Specimens: USNPC.

4. Syncuaria mycteriae Zhang, Brooks and Causey, 2003
Gizzard, Mycteria americana (A), Santa Rosa, Guanacaste (Zhang et al. 2003b). Specimens: USNPC.

5. Synhimantus (Dispharynx) nasuta Zhang, Brooks and Causey, 2004.

Craw, Thraupis episcopus (B), Estación San Gerardo, Guanacaste (Zhang et al. 2004a). Specimens: CHRC, USNPC.

Habronematidae (Chitwood y Wehr, 1932) Ivaschkin, 1961

6. Procyrnea mawsonae Zhang, Brooks and Causey, 2004

Craw, Buteo magnirostris (B), Cafetal, Guanacaste (Zhang et al. 2004b). Specimens: CHCR, USNPC.

7. $P$. maclennanae Zhang, Brooks and Causey, 2004

Craw, Heliomaster constantinii (B), Cafetal, Guanacaste (Zhang et al. 2004b). Specimens: CHCR, USNPC.

Hetarkidae

Railliet and Henry, 1912

8. Strongyluris panamensis Bursey, Goldberg and Telford, 2003

Intestine, Norops limifrons (R), Hacienda La Suerte, Limón (Bursey and Goldberg 2003). Specimens: HWML.

Onchocercidae (Leiper, 1911)

9. Litomosoides sp. ND, Artibeus jamaicensis (M), NA (Bain et al. 2002).

10. L. brasiliensis Almeida, 1936

Body cavity, Carollia perspicillata azteca (M), Santa Ana, San José (Bain et al. 2003). Specimens: CHCR.

Notes: Bain et al. (2003) identified this species based on fragments of females includes among type specimens of $L$. peñai.

11. Piratuba digiticauda Lent and Teixeira de Freitas, 1941

Body cavity, Norops limifrons (R), 
Hacienda La Suerte, Limón (Bursey and Goldberg 2003). Specimens: HWML.

Seuratidae (Hall, 1916)

$$
\text { Railliet, } 1906
$$

12. Skrjabinura mesamericana Zhang and Brooks, 2004.

Intestine, Calocitta formosa (B), Estación San Gerardo, Guanacaste (Zhang and Brooks, 2004). Specimens: CHCR, USNPC.

13. S. vali (Guerrero, 1971)

Caecum, Myiarchus tyrannulus, Piaya cayana (B), Cafetal, Guanacaste (Zhang and Brooks, 2004). Specimens: CHCR, USNPC.

\section{Taxonomic remarks on the status of some of the species of helminths from Costa Rica vertebrates}

Digenea (Van Beneden, 1858)

Cyclocoeliidae

Kossack, 1911

1. Uvitellina facioi (Brenes-Madrigal and Arroyo-Sancho, 1962)

Aerial sacs, Jacana spinosa (B), Aranjuez, Puntarenas (Brenes-Madrigal and ArroyoSancho 1962). Specimens: CHCR.

Notes: this species was originally described as Cyclocoelum facioi BrenesMadrigal and Arroyo-Sancho, 1962, included in the genus Haematotrephus Stossich, 1902 by Yamaguti (1971) and finally transferred to Uvitellina Witenberg, 1923 by Zamparo et al. (2003d).

Lecithodendriidae (Lühe, 1931)

Odhner, 1910

2. Langeronia macrocirra Caballero and Bravo-Hollis, 1949

Intestine, Bufo marinus and Rana warchewitschii $(\mathrm{N})$, Corís, Cartago (Brenes-
Madrigal et al. 1959). Specimens: CHCR. Notes: specimens originally determined as $L$. macrocirra, were transferred to $L$. provitellaria by Christian (1970) and later re-established as L. macrocirra by Martínez-Salazar (2004) who re-examined voucher specimens deposited in CHCR.

\section{Cestoidea}

Rudolphi, 1808

3. Mathevotaenia didelphidis (Rudolphi, 1819) Spasskii, 1951

Intestine, Marmosa sp. (M), Río Grande de Atenas, Alajuela (Flores-Barroeta et al. 1961).

Notes: this species was originally recorded as Oochoristica didelphidis (Rudolphi, 1819). Campbell et al. (2003) pointed out that these specimens differ from the original description of $M$. didelphidis and probably represent a distinct species.

4. M. pennsylvanica (Chandler and Melvin, 1951) Yamaguti, 1959

Intestine, Didelphis marsupialis etensis (M), Bello Horizonte, Escazú, San José (FloresBarroeta et al. 1958). Specimens: CNHE.

Notes: originally recorded as Oochoristica pennsylvanica Chandler and Melvin, 1951. Campbell et al. (2003) pointed out that description of specimens from Costa Rica differ from the original description of $M$. pennsylvanica in several traits.

NEMATODA (Rudolphi, 1808)

Onchocercidae (Leiper, 1911)

5. Litomosoides hamletti peñai (JiménezQuirós and Arroyo-Sancho, 1960)

Body cavity, Carollia perspicillata azteca (=Hemiderma perspicillata) (M), Santa Ana, San José (Jiménez-Quirós and Arroyo-Sancho 1960). Specimens: CHCR. Notes: Bain et al. (2003) analyzed the type material of Litomosoides peñai and proposed the subspecific status for this species. 


\section{Parasite-Host List}

Symbology: * new species; ${ }^{*}$ new genus; $\mathrm{A}=$ acanthocephalan; $\mathrm{D}=$ digenean; $\mathrm{N}=$ nematode.

\section{Reptiles}

Host

Basiliscus basiliscus

Ctenosaura quinquecarinata

Norops limifrons

Norops oxylophus

Host

Amazilia saucerrotei

Buteo magnirostris

Calocitta formosa

Caryothraustes poliogaster

Crypturellus cinnamomeus

Euphonia hirudinacea

Gymnopithys leucaspis

Heliomaster constantinii

Henicorhina leucosticta

Hylophylax naevioides

Jacana spinosa

Morococcyx erytrhopygius

Myiarchus nuttingi

Myiarchus tyrannulus

Mycteria americana

Phaenostictus mcleannani

Piaya cayana

Platyrhinchus coronatus

Thraupis episcopus

Thryothorus pleurostictus

Thryothorus nigricapillus

Tolmomyias sulphurescens
Helminth Species

Parallopharynx matternae* (D)

Parallopharynx gonzalezi (D)

Acanthocephalus saurius* (A)

Acuariidae (N)

Mesocoelium monas (D)

Piratuba digiticauda (N)

Strongyluris panamensis $(\mathrm{N})$

Urotrema shirleyae* (D)

\section{Aves}

Helminth Species

Whallwachsia illuminata $*$ (D)

Procyrnea mawsonae* $(\mathrm{N})$

Skrjabinura mesamericana* $(\mathrm{N})$

Pojmanskia riosae (D)

Tinamutrema canoae ** (D)

Pojmanskia riosae (D)

Bakkeius moragai $* \ddagger$ (D)

Acuaria wangi $(\mathrm{N})$

Procyrnea maclennanae* $(\mathrm{N})$

Bakkeius moragai (D)

Acuaria wangi* $(\mathrm{N})$

Neohaematotrephus arayae* (D)

Pojmanskia riosae (D)

Acuaria mayori $(\mathrm{N})$

Skrjabinura vali $(\mathrm{N})$

Syncuaria mycteriae* $(\mathrm{N})$

Bakkeius moragai (D)

Skrjabinura vali (N)

Pojmanskia riosae (D)

Synhimantus (Dispharynx) nasuta*(N)

Bakkeius moragai (D)

Pojmanskia riosae* ${ }^{* \#}$ (D)

Pojmanskia riosae (D)

Whallwachsia illuminata (D)

\section{Mammalia}

Host

Artibeus jamaicensis

Carollia perspicillata azteca ${ }^{* *}$
Helminth Species

Litomosoides sp. (N)

Litomosoides brasiliensis $(N)$

** recorded as Hemiderma perspicillatum in the original study 


\section{DISCUSSION}

After June, 2002, when we finished the compilation of the original "Checklist of the helminth parasites of vertebrates in Costa Rica” (Rodríguez-Ortíz et al. 2004), 14 papers have been published with new records on the helminth fauna of Costa Rican vertebrates (particularly in the Área de Conservación de Guanacaste). In these studies, 23 additional species of helminths parasitizing 25 species of vertebrates have been described. About $76 \%$ of these new material was deposited in the CHCR. This fact establish a new era for the Colección de Helmintos de Costa Rica, since this collection will become a primary repository for parasite biodiversity in the country. To summarize, up to date 325 helminth species have been described in vertebrates from Costa Rica, represented by 89 species of digeneans, 23 of monogeneans, 63 of cestodes, 13 of acanthocephalans, and 137 of nematodes. We encourage people describing helminth parasites of this country to send specimens to be deposited and saved in this re-emerged collection.

\section{ACKNOWLEDGMENTS}

To Daniel R. Brooks for providing information form their databases. Funds to complete this paper were provided by the agreement between the Universidad de Costa Rica and the Instituto Mexicano de Cooperación Internacional through the project: "Support to catalog and maintain the Helminthological collection of the University of Costa Rica".

\section{RESUMEN}

En este trabajo presentamos una addenda al "Listado de helmintos parásitos de vertebrados de Costa Rica". Esta addenda actualiza la información disponible acerca de este grupo de parásitos en dicho país, cuyo estudio recientemente ha tomado un nuevo impulso. En este trabajo, se adicionan 33 registros, representando 23 especies. Lo anterior eleva el número de especies de helmintos parásitos de vertebrados de Costa Rica registrado hasta ahora a 325: 89 especies de digéneos, 23 de monogéneos, 63 de céstodos, 13 de acantocéfalos, y 137 de nemátodos. En total, 133 especies de vertebrados han sido estudiadas en busca de helmintos en Costa Rica (31 especies de peces, 7 de anfibios, 18 de reptiles, 40 de aves, y 37 de mamíferos). Hasta la fecha, 67 especies (20.6\%) fueron descritas como nuevas en Costa Rica y muchas de ellas son endémicas a una región particular. La Colección de Helmintos de Costa Rica, localizada en la Universidad de Costa Rica (UCR) en San José, resguarda material de únicamente el 23\% de las especies registradas para el país, pero afortunadamente esto está cambiando actualmente.

\section{REFERENCES}

Bain, O., S. Babayan, J. Gomes, G. Rojas \& R. Guerrero. 2002. First account on the larval biology of a Litomosoides filaria, from a bat. Parassitol. 44: 89-92.

Bain, O., R. Guerrero, B. Rodríguez, S. Babayan \& N. Jouvenet. 2003. Examination of type material of two species of Litomosoides (Filaroidea: Onchocercidae), parasites from bats; taxonomic consequences. Parasite. 10: 211-218.

Brenes-Madrigal, R. \& G. Arroyo-Sancho. 1962. Helmintos de la República de Costa Rica XX. Algunos tremátodos de aves silvestres. Rev. Biol. Trop. 10: 205-227.

Brenes-Madrigal, R., G. Arroyo-Sancho \& E. Delgado-Flores. 1959. Helmintos de la República de Costa Rica XI. Sobre la validez del género Langeronia Caballero y Bravo, 1949 (Trematoda: Lecithodendriidae) y hallazgo de Ochetosoma miladelarocai Caballero y Vogelsang, 1947. Rev. Biol. Trop. 7: 81-87.

Bursey, C.R. \& S.R. Goldberg. 2003. Acanthocephalus saurius $\mathrm{n}$. sp., (Acanthocephala: Echinorhynchidae) and other helminths from the lizard Norops limifrons (Sauria: Polychrotidae) from Costa Rica. J. Parasitol. 89: $573-576$

Caballero, C.E. \& R. Brenes-Madrigal. 1958. Helmintos de la República de Costa Rica VII. Tremátodos de algunos vertebrados salvajes, con descripción de una nueva especie de Acanthostomum Looss, 1899. An. Inst. Biol. Univ. Nac. Autón. Méx. 29: 165-179.

Campbell, M.L., S.L. Gardner \& G.T. Navone. 2003. A new species of Mathevotaenia (Cestoda: Anoplocephalidae) and other tapeworms from marsupials in Argentina. J. Parasitol. 89: 1181-1185.

Christian, A.F. 1970. Langeronia parva sp. n. (Trematoda: Lecithodendriidae) with revision of the genus Langeronia Caballero and Bravo-Hollis, 1949. J. Parasitol. 56: 321-324. 
Flores-Barroeta, L., E. Hidalgo-Escalante \& R. BrenesMadrigal. 1958. Céstodos de Vertebrados IV. Rev. Biol. Trop. 6: 55-78.

Flores-Barroeta, L., E. Hidalgo-Escalante \& F. MonteroGei. 1961. Céstodos de vertebrados. VIII. Rev. Biol. Trop. 9: 187-207.

Jiménez-Quirós, O. \& G. Arroyo-Sancho. 1960. Helmintos de la República de Costa Rica. XV. Nematoda 3. Presencia de Litomosoides peñai $\mathrm{n}$. sp. en Hemiderma perspicillata aztecum (Saussure, 1860). Rev. Biol. Trop. 8: 63-67.

Martínez-Salazar, E.A. 2004. Estudio taxonómico de algunas poblaciones del género Langeronia Caballero y Bravo-Hollis, 1949 (Trematoda: Lecithodendriidae) en México. Tesis de Maestría, Universidad Nacional Autónoma de México, México D.F., México.

Rodríguez-Ortiz, B., L. García-Prieto \& G. Pérez-Ponce de León. 2004. Checklist of the helminth parasites of vertebrates in Costa Rica. Rev. Biol.Trop. 52 (Supl.1): 1-41.

Teixeira de Freitas, J. F. 1963. Revisao de Familia Mesocoelidae Dollfus, 1933. Mem. Inst. Oswaldo Cruz. 61: 177-311.

Zamparo, D., D.R. Brooks \& D. Causey. 2003a. Tinamutrema canoae n. gen. n. sp. (Trematoda: Digenea: Strigeiformes: Brachylaimidae) in Crypturellus cinnamomeus (Aves, Passeriformes, Tinamidae) from the Area de Conservación Guanacaste, Costa Rica. J. Parasitol. 89: 819-822.

Zamparo, D., D.R. Brooks \& D. Causey. 2003b. Bakkeius moragai n. gen. n. sp. and Pojmanskia riosae n. gen. n. sp. (Trematoda: Digenea: Brachylaimoidea) in birds from the Area de Conservación Guanacaste, Costa Rica. J. Parasitol. 89: 823-828.

Zamparo, D., D.R. Brooks \& D. Causey. 2003c. Wallwachsia illuminata $\mathrm{n}$. gen., n. sp. (Trematoda: Digenea: Plagiorchiformes: Prosthogonimidae) in the steelyvented hummingbird Amazilia saucerrottei (Aves: Apodiformes: Trochilidae) and the yellow-olive flycatcher Tolmomyias sulphurescens (Aves: Passeriformes: Tyraninidae) from the Area de Conservación Guanacaste, Guanacaste, Costa Rica. J. Parasitol. 89: 814-818.
Zamparo, D., D.R. Brooks, D. Causey \& B. Rodríguez. 2003d. Neohaematotrephus arayae n. sp. (Digenea: Echinostomiformes: Cyclocoelidae) in Jacana spinosa (Aves: Charadriiformes: Jacanidae) from the Area de Conservación Guanacaste, Costa Rica. J. Parasitol. 89: 829-831.

Zamparo, D., D.R. Brooks \& V. Tkach. 2004a. A new species of Urotrema (Digenea: Plagiorchiformes: Urotrematidae) in Norops oxylophus (Squamata) from the Area de Conservación Guanacaste, Costa Rica. J. Parasitol. 90: in press.

Zamparo, D., D.R. Brooks, V. Tkach \& B. Rodriguez. 2004b. Parallopharynx matternae n. sp. (Trematoda: Digenea: Plagiorchiidae) in Basiliscus basiliscus (Squamata: Iguania: Corytophanidae) from the Area de Conservación Guanacaste, Guanacaste, Costa Rica. J. Parasitol. 90: in press.

Zhang, L. \& D.R. Brooks. 2004. Skrjabinura mesoamericana $\mathrm{n}$. sp. (Nematoda: Seuratoidea: Seuratidae) in birds from the Area de Conservación Guanacaste, Costa Rica. J. Parasitol. 90: in press.

Zhang, L., D.R. Brooks \& D. Causey. 2003a. Two species of Acuaria Bremser, 1811 (Nematoda: Acuarioidea: Acuariidae) in Passerine birds from the Area de Conservación Guanacaste, Costa Rica. J. Parasitol. 89: 1039-1043.

Zhang, L., D.R. Brooks \& D. Causey. 2003b. A new species of Syncuaria Gilbert, 1927 (Nematoda: Acuarioidea: Acuariidae) in the wood stork, Mycteria americana L. (Aves: Ciconiiformes: Ciconiidae) from the Area de Conservación Guanacaste, Costa Rica. J. Parasitol. 89: 1034-1038.

Zhang, L., D.R. Brooks \& D. Causey. 2004a. Two species of Synhimantus (Dispharynx) Railliet, Henry and Sisoff, 1912 (Nematoda: Acuarioidea: Acuariidae) in passerine birds from the Area de Conservación Guanacaste, Costa Rica. J. Parasitol. 90: in press.

Zhang, L., D.R. Brooks \& D. Causey. 2004b. Procyrnea Chabaud, 1958 (Nematoda: Habronematoidea: Habronematidae) in birds from the Area de Conservación Guanacaste, Costa Rica. J. Parasitol. 90: in press.

Yamaguti, S. 1971. Synopsis of Digenetic Trematodes of Vertebrates. Keigaku Publishers, Tokyo. 1074 p. 
